\title{
Insulin modulates the secretion of proteins from mature 3T3-L1 adipocytes: a role for transcriptional regulation of processing
}

\author{
P. Wang $\cdot$ J. Keijer $\cdot$ A. Bunschoten $\cdot$ F. Bouwman • \\ J. Renes $\cdot$ E. Mariman
}

Received: 1 March 2006 / Accepted: 19 April 2006 / Published online: 4 August 2006

(C) Springer-Verlag 2006

\begin{abstract}
Aims/hypothesis Under conditions of insulin resistance and type 2 diabetes, fat cells are subjected to increased levels of insulin, which may have a major impact on the secretion of adipokines.

Materials and methods Using transcriptomics and proteomics, we investigated how insulin affects the transcription and protein secretion profile of mature 3T3-L1 adipocytes. Results We found that insulin has a significant impact on protein secretion of 3T3-L1 adipocytes. However, transcription is not the major regulation point for these secreted proteins. For extracellular matrix components, our data suggest that the mRNA level of processing enzymes, but not of target proteins, is the regulating point at which insulin stimulates secretion and function of the relevant proteins. Among these enzymes, we report a novel finding, namely that sulfatase 2 gene is regulated by insulin, which may induce a functional change in cultured adipocytes.

Conclusions/interpretation We propose that enhancement of protein processing and secretion rather than transcription

Electronic supplementary material Supplementary material is available for this article at http://dx.doi.org/10.1007/s00125-0060321-5 and is accessible for authorized users.

P. Wang $\cdot$ F. Bouwman $\cdot$ J. Renes $\cdot$ E. Mariman

Functional Genomics Group, Research Institute NUTRIM,

Department of Human Biology, Maastricht University,

Maastricht, The Netherlands

P. Wang $\cdot$ J. Keijer $\cdot$ A. Bunschoten

Food Bioactives Group, Rikilt-Institute of Food Safety,

Wageningen, The Netherlands

E. Mariman $(\triangle)$

Department of Human Biology, Maastricht University,

P.O. Box 616, 6200 MD Maastricht, The Netherlands

e-mail: E.Mariman@HB.unimaas.nl
\end{abstract}

of the secreted protein genes is part of the strategic role of insulin in the induction of cellular responses.

Keywords Adipocytes · Insulin · Proteomics · Secretion · Transcriptomics
Abbreviations
1D one-dimensional
2D two-dimensional
ECM extracellular matrix
ESM Electronic Supplementary Material
Glc low insulin high glucose condition
HSPG heparan sulfate proteoglycans
Ins high insulin low glucose condition
Ins+ Glc high insulin high glucose condition
MALDI-TOF matrix assisted laser desorption/ionisation-time
P4ha1 procollagen-proline, 2-oxoglutarate 4-dioxy-
genase (proline 4-hydroxylase), alpha 1
Pcolce procollagen C-endopeptidase enhancer protein
PTM post-translational modification
Q-PCR quantitative real time RT-PCR analysis
SPARC secreted acidic, cysteine-rich protein
Sulf $2 \quad$ sulfatase 2

\section{Introduction}

Obesity is associated with insulin resistance and type 2 diabetes, implying that adipose tissue plays a role in the development of these disorders [1]. The primary function of adipose tissue is storage of fat, but it is also recognised as an active regulation centre, providing signals to guide metabolism. Well-known examples of signalling molecules 
(adipokines) secreted by adipose tissue are leptin, adiponectin, resistin and IL-6 [2]. Adipokine secretion is responsive to afferent messages to the fat cells [3]. Under conditions of insulin resistance and type 2 diabetes, fat cells are subjected to increased levels of insulin, which may have a major impact on the adipokines. A number of reviews have provided overviews of the relation between circulating levels of insulin and specific adipokines in rodents and humans [2, 4]. According to these, IL-6 and leptin have shown a consistently positive relation with insulin in various studies, while the response of resistin and adiponectin to insulin remains contradictory.

Often a high level of insulin is associated with hyperglycaemia. Only in the first stage of insulin resistance or at the later stage of type 2 diabetes do high insulin and high glucose not appear in parallel. As insulin targets, adipocytes respond to insulin by enhancing glucose uptake, which is impaired in insulin resistance and type 2 diabetes [5]. High levels of glucose alone may induce the production of inflammatory factors and acutephase reactants in adipocytes [6]. However, in in vivo investigations of obesity and type 2 diabetes, the contributions from high insulin and/or high glucose were hardly distinguished [2].

The effect of insulin on protein secretion has been addressed specifically in vitro using mouse 3T3-L1 adipocytes. It was shown that insulin can stimulate exocytosis of a regulated secretory compartment containing adiponectin, which is distinct from that containing type VI alpha 3 collagen [7]. Another study indicated that insulin stimulates the activity of the constitutive secretory pathway, possibly by increasing the activity of phospholipase D [8]. Despite the information obtained, these studies also showed that the mechanism of protein secretion regulation by insulin in adipocytes is complex and warrants further detailed study.

'Omics' technology provides powerful tools for expression profiling of biological events. Numerous studies have used genomics-approaches to investigate insulin actions in relation to insulin resistance and type 2 diabetes, but only few specifically addressed the effect of insulin on adipocyte secretion [9]. In our previous work we set up a twodimensional (2D) gel-secreted protein map of mouse 3T3L1 adipocytes [10]. In addition to lipid metabolism regulators and growth factors, we found that the majority of secreted proteins belong to extracellular components and their related proteins. Here, using transcriptomics and proteomics, we investigated how insulin affects the transcription and protein secretion profile of mature 3T3-L1 adipocytes. As insulin stimulates glucose uptake and differences in intracellular glucose levels may affect gene expression, we performed our studies under conditions of low and high glucose.

\section{Materials and methods}

Cell culture and sample preparation

Murine 3T3-L1 fibroblasts (American Type Culture Collection, ATCC Number: CL-173) were cultured and differentiated to adipocytes in vitro as previously described [10], except that in the adipogenic cocktail $1 \mu \mathrm{mol} / 1$ 15deoxy- $\Delta^{12,14}$-prostaglandin $\mathrm{J}_{2}$ (Cayman Chemical, Ann Arbor, MI, USA) was used instead of $10 \mu \mathrm{mol} / 1$ prostaglandin $\mathrm{I}_{2}$ (Carbacyclin). On day 8, differentiated cells grown in DMEM/F-12 (1:1) medium supplemented with 10\% fetal calf serum (Perbio Science, Erembodegem, Belgium) were treated with $1 \mu \mathrm{mol} / \mathrm{l}$ insulin (cat. no. I 1882; Sigma, Zwijndrecht, the Netherlands) and $4.5 \mathrm{mmol} / \mathrm{l}$ glucose (high insulin low glucose condition [Ins]) or $15.75 \mathrm{mmol} / 1$ glucose (high insulin high glucose condition [Ins+Glc]). In parallel, control cells were cultured in the same medium with $4.5 \mathrm{mmol} / 1$ glucose alone (Basal) or $15.75 \mathrm{mmol} / 1$ glucose alone (low insulin high glucose condition, [Glc]). Each condition had quadruplicates. The Ins vs basal conditions were used to monitor the effect of insulin at the low glucose level; the Ins+Glc vs Glc conditions were used to do the same at the high glucose level. On day 10, two replications with approximately $2.6 \times 10^{6}$ cells of all conditions were collected separately for total RNA isolation using Trizol (Invitrogen, Breda, the Netherlands) according to the manufacturer's instruction, and after chloroform extraction, an extraction of phenol: chloroform: isoamylalcohol (25:24:1), followed by a chloroform extraction, was added. Quality and quantity of total RNA were checked by spectrophotometer and agarose gel. Cells of the two other replications with approximately $7 \times 10^{6}$ cells were washed and incubated with the corresponding serum-free medium, supplemented with $2.2 \mathrm{mg} / 1$ transferrin and $10.6 \mathrm{nmol} / 1$ sodium selenite, for $6 \mathrm{~h}$. The number of cells was counted and culture medium proteins were collected, dialysed, lyophilised and dissolved as described before [10]. The duplicated protein samples were pooled for later analysis. Three independent experiments were performed.

\section{Microarray}

The mouse 50-mer 10K_A oligo set (MWG, Ebersberg, Germany) and additional 50-mer oligonucleotides of genes encoding secretory proteins and proteins involved in energy metabolism were printed in-house on GAP II-coated glasses (Corning, Acton, MA, US) using a Microgrid II arrayer (BioRobotics, Cambridge, UK). A total of 10,117 (10,060 non-redundant) mouse oligo probes was arrayed. In addition to the manufacturer's annotation, the oligonucleotides were further annotated with Gene Ontology using SOURCE 
http://smd.stanford.edu/cgi-bin/source/sourceBatchSearch, last accessed 28 October 2005).

We used five total RNA samples of three independent experiments per condition for hybridisation of the oligo DNA arrays as described [11]. For this, total RNA samples were reverse transcribed (45 $\mu \mathrm{g}$ scale) to cDNA and labelled with Cy5 using a direct labelling method as described [12]. A pool of total RNA from all samples labelled with $\mathrm{Cy} 3$ was used as a 1:1 (v/v) reference in the hybridisations. The signals were captured by ScanArray Express HT (PerkinElmer Life Sciences, Billerica, MA, US) at $90 \%$ laser power and 60\% photomultiplier tube voltage.

\section{Microarray data analysis}

The data were extracted (medium density) using ArrayVision (version 7.0, Imaging Research, St Catharines, ON, Canada). The overall quality of the slides was checked both by visual inspection and Bland-Altmen plots. Next, the eligible array data were exported into GeneMaths XT (Applied Maths, Sint-Martens-Latem, Belgium). Spots with an average intensity, over all arrays, of lower than two-fold above average background were removed from further analysis. Then, the Cy5 intensities of the remaining 4,019 spots were normalised against the $\mathrm{Cy} 3$ reference exactly as described [11]. The differences between samples were visualised using GeneMaths embedded discriminant analysis. This is a supervised form of principal component analysis, based on all expression data, and in which samples are first assigned to their treatment group and then separated along the dimension of maximum variation in gene or protein expression between the groups. Individual spots with a signal more than two-fold different from the average of the replicates $(n=5)$ were excluded and flagged. Fold change calculations and Student $t$-tests were performed in Microsoft Excel. Fold change equals ratio in the case of increase or equals -1 / ratio in the case of decrease. Cut-off criteria for differential expression was set at a fold change of $>1.3$ and $p<0.05$. For genes with replicated spots, the average value of individual spots was used.

\section{Quantitative real-time RT-PCR}

All six total RNA samples per treatment were used in quantitative real-time RT-PCR analysis (Q-PCR) of 18 genes (primer information, see Electronic Supplementary Material [ESM] Table 1). After cDNA synthesis using an iScript kit (Bio-Rad, Veenendaal, the Netherlands), Q-PCR was conducted in iQ SYBR Green Supermix (MyiQ SingleColor Real-Time PCR Detection System; Bio-Rad), according to the manufacturer's instructions. The standard curve method was used in Q-PCR data analysis. Ribosomal protein S15 was selected as reference gene on the basis of its constant expression behaviour on the array.

One- and two-dimensional gel electrophoresis

Protein samples obtained from $2.4 \times 10^{6}$ cells of each condition were analysed by 2D gel electrophoresis as described [10]. The data were obtained from PDQuest (version 7.2; Bio-Rad) and the gel density was normalised on the basis of total quantity in a spiked set of molecular weight markers. The gels were matched to a secreted protein reference map for 3T3-L1 adipocytes [10] and 99 secreted spots of $<85 \mathrm{kDa}$ were matched. The data were exported into GeneMaths for discriminant principal component analysis. A 1.8-fold change was taken as cut-off for regulation. Protein samples obtained from $2.2 \times 10^{5}$ cells were separated using 18-cm one-dimensional (1D) SDSPAGE (Protean II XL; Bio-Rad) to analyse high-molecularweight proteins. After silver staining [10], gel images were obtained with a GS-800 calibrated densitometer and analysed with Quantity One (Bio-Rad). An adipocyte medium protein sample treated with Brefeldin A [10] was loaded on the same gel, to verify protein secretion. Protein bands were excised and identified by matrix assisted laser desorption/ionisation-time of flight (MALDI-TOF) mass spectrometry [13].

\section{Results}

Insulin generates minor changes in the transcriptome of mature adipocytes in vitro

To investigate the effect of insulin on mature adipocytes, 3T3-L1 adipocytes were cultured for 2 days with $1 \mu \mathrm{mol} / 1$ insulin with either high glucose or low glucose. For reference, cells were cultured with high or low glucose only. The morphology of the adipocytes as examined by light microscopy was similar for all four conditions. Total RNA samples from three independent experiments, performed in duplicate for each of the four conditions, were individually hybridised against a common reference pool on oligo DNA arrays. Two arrays were excluded from the analysis for quality reasons, one for Ins+Glc and the other for Ins. A total of 4,019 of 10,117 probes with average signals higher than two-fold over the background signal and representing 3,718 non-redundant genes were used for further data analysis. The comparison between the insulin and non-insulin treatment at the same glucose level showed minor changes in the transcriptome. No gene changed $>2$ fold in either comparison. Of the probe signals, 92.4\% (low glucose) or $90.4 \%$ (high glucose) were in the range of -1.2to 1.2 -fold. 
Using a fold change of 1.3 and a $p$ of 0.05 as cut-off, 105 genes were found to be differentially expressed in mature adipocytes by insulin treatment at low or high glucose levels (Fig. 1). The overlap of the insulin-regulated gene list between low and high glucose levels was small, indicating an influence of glucose on the effect of insulin. We used Q-PCR to confirm the mRNA change for 12 of the differentially expressed genes and six of their family members. A good correlation between array and Q-PCR data was found $\left(R^{2}=0.803\right)$. Three examples, procollagenproline, 2-oxoglutarate 4-dioxygenase (proline 4-hydroxylase), alpha 1 (P4hal), procollagen C-endopeptidase enhancer protein (Pcolce) and sulfatase 2 (Sulf2), are shown (Fig. 2).

The 105 differentially expressed genes were clustered into ten functional groups based on Gene Ontology annotations (Fig. 3, ESM Table 2). One additional group consisted of genes with unknown function. The largest group was that of structural proteins and related enzymes, consisting of genes with a functional annotation of cytoskeleton, cell adhesion and extracellular matrix (ECM) (Table 1). All genes of this functional group were upregulated, except cysteine-rich protein 61. Sulf2, an extracellular endosulfatase, showed the largest increase in response to insulin (1.8-fold). This was not influenced by the glucose state. Glucose alone had no effect on its expression (Table 1, Fig. 2). The up-regulation of proteolysis inhibitors, tissue inhibitor of metalloproteinases (Timp1 and Timp4), implies reduced degradation of ECM components. The next major functional group was related to protein expression. However, the upregulation or downregulation of individual genes involved in mRNA processing and protein synthesis, folding, modification and transport gave an inconsistent picture of the effect of insulin on protein expression in general (ESM Table 2). For all groups the insulin effect was more explicit with high glucose than

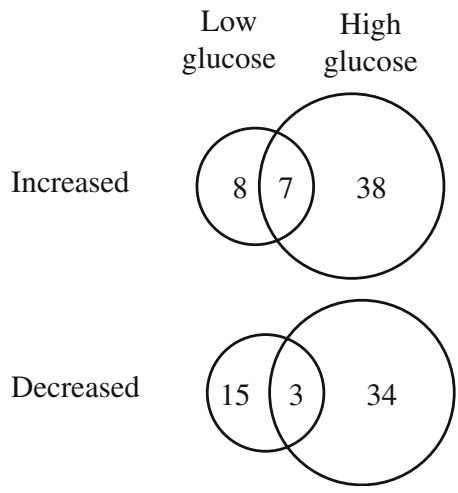

Fig. 1 Number of genes regulated by insulin at low and high glucose level. Based on microarray data, insulin and basal conditions were compared to obtain the number of regulated genes for the low glucose level; insulin+glucose and glucose conditions were compared for the high glucose level. Cut-off criteria: fold change $>1.3$ and $t$-test $p<0.05$ with low glucose, while glucose itself had no direct effect on the expression of most of these genes. This implies that regulation of these processes by insulin is dependent on the level of glucose.

We analysed the expression of 38 genes on the array and involved in insulin signalling pathways and found no significant effect (ESM Table 3). Since insulin signal transmission occurs mostly via phosphorylation of the proteins, it is not surprising that our data showed no significant influence on mRNA levels. One exception was phosphatidylinositol 3-kinase, regulatory subunit, polypeptide 2 (p85 beta), which was upregulated by insulin (fold change $=1.22, p=0.046$ and fold change $=1.39, p=0.011$ at low and high glucose, respectively). It has been reported that p85 beta negatively regulates the downstream signalling of phosphatidylinositol 3-kinase [14]. Therefore, the up-regulation of p85 beta suggests an attenuation of particular insulin signalling pathways.

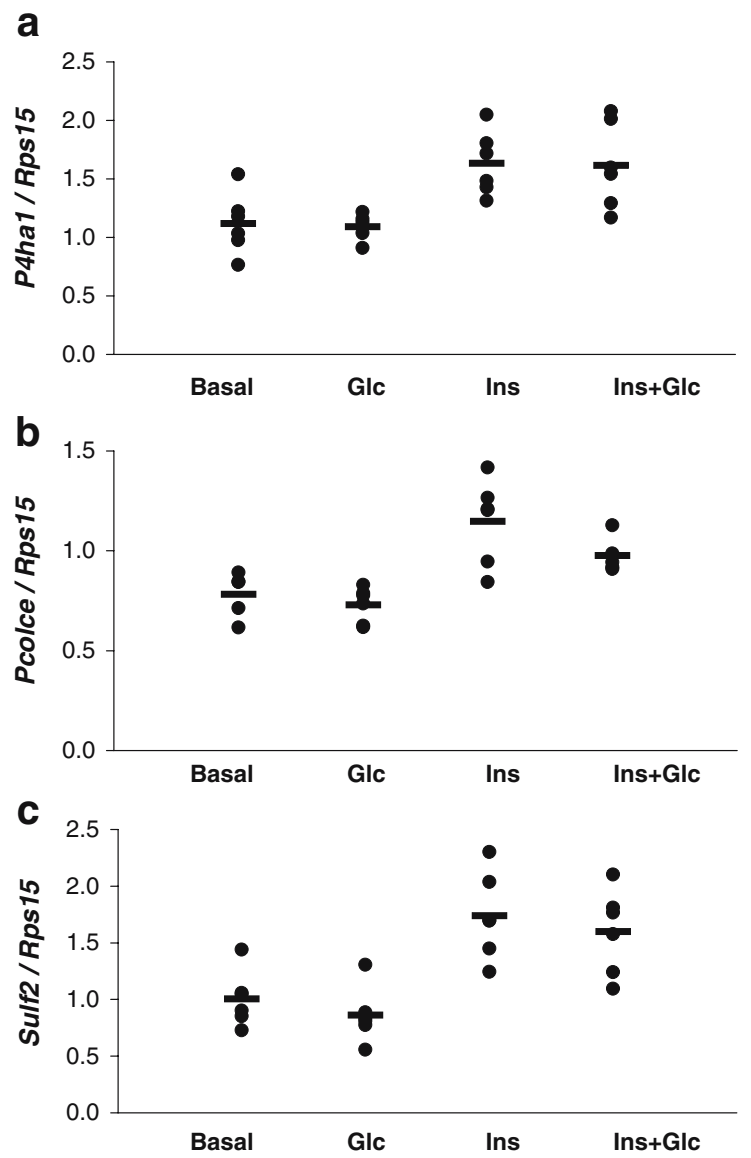

Fig. 2 Expression levels of genes involved in protein processing measured by quantitative real-time PCR, relative to ribosomal protein S15 (Rps15) expression. Dots, individual samples; bars, group average $(n=6)$. The fold change of Ins/Basal and Ins $+\mathrm{Glc} / \mathrm{Glc}$, respectively, were for: (a) P4hal $1.46(p=0.008)$ and $1.48(p=0.016)$; (b) Pcolce $1.47(p=0.007)$ and $1.34(p=0.001)$; and (c) Sulf2 $1.73(p=$ $0.004)$ and $1.86(p=0.003)$ 


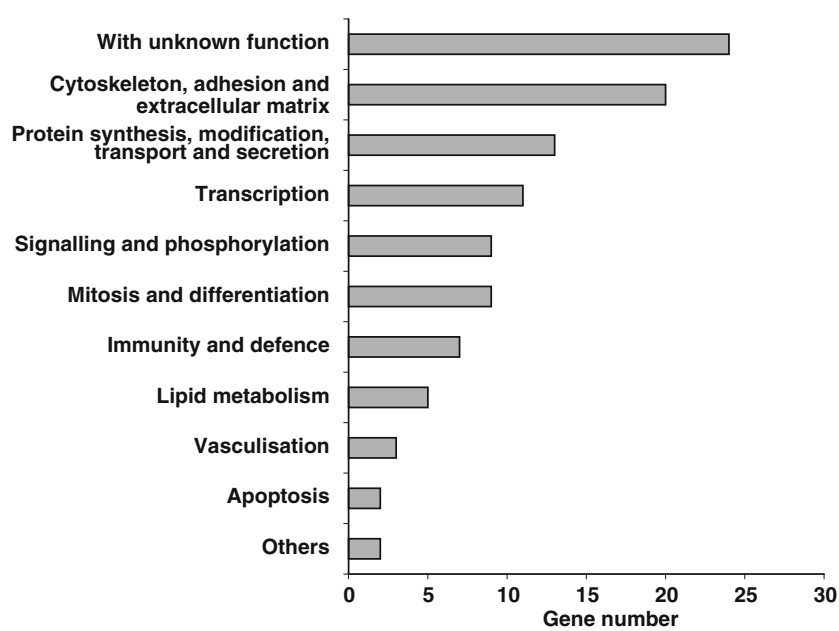

Fig. 3 Functional categories of genes regulated by insulin

Insulin modulates protein secretion from mature adipocytes in vitro

No clear effect of insulin was seen on the expression of genes encoding secreted proteins or proteins involved in secretion pathways. To assess whether this would also be the case at the protein level, we also analysed the secreted proteins of an identical set of experiments performed in parallel to those described for mRNA expression analysis using 2D gel electrophoresis. Using a secretory protein map of 3T3-L1 adipocytes [10], we identified 86 secreted spots corresponding to 37 genes in the 10 to $85 \mathrm{kDa}$ range. Highmolecular-weight proteins were left out of the analysis due to overall low resolution in the high-molecular-weight part of the gel. A strong effect of insulin on the expression of secreted proteins was detected. A total of 63 spots $(73.3 \%)$ corresponding to 29 genes $(78.4 \%)$ were upregulated or downregulated by insulin at either high or low glucose level. The majority of the secreted proteins were affected at both high and low glucose levels (Table 2). The most dominant change in the secretion profile was the increase of the C-peptides of collagen type I and III, which are the processing by-products of fibrillar collagens (Fig. 4).

To obtain information on the high-molecular weight secretion profile, we used 1D gel electrophoresis. Three bands that showed differential expression and were verified as secreted by brefeldin A [10] were identified by MALDITOF mass spectrometry. They contain ECM components (procollagens and nidogen) and whole-chain complement C3 (Fig. 5).

The combined secretion profiles showed that insulin increases the secretion and processing of ECM components, such as collagen type I, III, V, VI and nidogen. The upregulation of ECM processing is in line with the increased secretion of processing enzymes and their regulators, such as matrix metalloproteinase 2 , metalloproteinase inhibitor
2 and procollagen $\mathrm{C}$-endopeptidase enhancer protein (Table 2). The secretion of most of the detected adipokines, such as complement $\mathrm{C} 3$, adipsin, secreted acidic cysteinerich protein (SPARC), and pigment epithelium-derived factor, was stimulated by insulin. The downregulated secretory spots (Table 2) were all from intracellular proteins [10], except adiponectin and galectin-1. Western blotting confirmed that adiponectin was not upregulated by insulin, but this technique also failed to show any significant downregulation (data not shown).

Comparison of insulin effect on transcriptome and proteome of secretory genes

Discriminant principal component analysis is a powerful tool for illustrating the general effect of different treatments. Discriminant principal component analysis of the $2 \mathrm{D}$ secreted protein profile confirmed that, compared with glucose alone, insulin generated a clear shift of the secreted protein profile (Fig. 6a). In contrast, the transcription profile of the 36 corresponding genes (nascent polypeptideassociated complex alpha polypeptide gene was not included due to its low expression) was hardly affected (Fig. 6b).

The discrepancy between the effect of insulin at the secreted protein level and the mRNA level as observed by discriminant principal component analysis was verified by zooming in on individual proteins/genes (Table 2). Of the 37 genes, 29 were changed by insulin treatment at the secreted protein level. However, only four of them (superoxide dismutase 3 extracellular, macrophage migration inhibitory factor, procollagen type $\mathrm{V}$ alpha 1 and Pcolce) showed a comparable regulation at mRNA level, while the others were not regulated at the transcriptional level.

The strong increase of collagen C-peptides indicated considerable regulation at the protein processing level. In this respect, we found two interesting genes. Prolyl 4hydroxylase catalyses the first post-translational modification (PTM) of collagen, resulting in the 4-hydroxylation of a number of proline residues [15]. Active prolyl 4-hydroxylase is a tetramer composed of two pairs of non-identical subunits $(\alpha 2 \beta 2)$. The concentration of alpha-subunit limits the rate of formation of active enzyme [16]. Microarray data, confirmed by Q-PCR, showed that the expression of $P 4 h a 1$ was significantly upregulated by insulin at both low and high glucose levels (Fig. 2). Glucose itself had no effect on this gene, indicating that this upregulation is glucose-independent and insulin-specific. Cleavage of $\mathrm{N}$ and C-peptides is also an important step in fibrillar collagen maturation. The $\mathrm{C}$-endopeptidase enhancer protein (PCOLCE) binds and drives the enzymatic cleavage of type I procollagen [17]. Although the C-endopeptidase 
Table 1 Expression of cytoskeleton, adhesion and extracellular matrix genes regulated by insulin

\begin{tabular}{|c|c|c|c|c|c|c|c|c|c|}
\hline \multirow[t]{2}{*}{ Function } & \multirow[t]{2}{*}{ Symbol } & \multirow[t]{2}{*}{ GeneID } & \multirow[t]{2}{*}{ Name } & \multicolumn{2}{|c|}{ Ins/basal } & \multicolumn{2}{|c|}{ Ins + Glc/Glc } & \multicolumn{2}{|c|}{ Glc/basal } \\
\hline & & & & $\mathrm{FC}$ & $p$ & $\mathrm{FC}$ & $p$ & $\mathrm{FC}$ & $p$ \\
\hline \multicolumn{10}{|l|}{ Cytoskeleton } \\
\hline & Tubb6 & 67951 & Tubulin, beta 6 & 1.18 & 0.302 & 1.36 & 0.025 & -1.14 & 0.230 \\
\hline Actin binding & Cnn3 & 71994 & Calponin 3 , acidic & 1.30 & 0.007 & 1.29 & 0.018 & -1.10 & 0.230 \\
\hline Actin binding & $\operatorname{Tag} \ln 2$ & 21346 & Transgelin 2 & 1.40 & 0.033 & 1.45 & 0.011 & -1.13 & 0.336 \\
\hline \multicolumn{10}{|l|}{ Adhesion } \\
\hline & Postn & 50706 & Periostin, osteoblast specific factor & 1.32 & 0.049 & 1.38 & 0.044 & -1.23 & 0.170 \\
\hline & $\operatorname{Itga} 5$ & 16402 & Integrin alpha 5 & 1.11 & 0.365 & 1.42 & 0.001 & -1.28 & 0.061 \\
\hline & Cyr61 & 16007 & Cysteine-rich protein 61 & -1.01 & 0.880 & -1.41 & 0.035 & 1.38 & 0.035 \\
\hline & $S d c 1$ & 20969 & Syndecan 1 & 1.22 & 0.187 & 1.51 & 0.006 & -1.29 & 0.091 \\
\hline & Cspg2 & 13003 & Chondroitin sulfate proteoglycan 2 & 1.51 & 0.002 & 1.33 & 0.198 & 1.11 & 0.315 \\
\hline $\begin{array}{l}\text { Proteoglycan } \\
\text { synthesis }\end{array}$ & Chst2 & 54371 & Carbohydrate sulfotransferase 2 & 1.39 & 0.013 & 1.31 & 0.045 & -1.13 & 0.240 \\
\hline $\begin{array}{c}\text { Proteoglycan } \\
\text { hydrolysis }\end{array}$ & Sulf2 & 72043 & Sulfatase 2 & 1.76 & 0.013 & 1.73 & 0.009 & -1.02 & 0.804 \\
\hline \multicolumn{10}{|l|}{ Extracellular matrix } \\
\hline & $T g f b i$ & 21810 & Transforming growth factor, beta induced & 1.35 & 0.005 & 1.12 & 0.024 & -1.18 & 0.012 \\
\hline & Col5al & 12831 & Procollagen, type $\mathrm{V}$, alpha 1 & 1.11 & 0.290 & 1.32 & 0.018 & -1.10 & 0.334 \\
\hline & Col6a3 & 12835 & Procollagen, type VI, alpha 3 & 1.25 & 0.001 & 1.47 & 0.009 & -1.09 & 0.131 \\
\hline & Col6a2 & 12834 & Procollagen, type VI, alpha 2 & 1.11 & 0.116 & 1.31 & 0.029 & -1.06 & 0.401 \\
\hline & Col5a3 & 53867 & Procollagen, type V, alpha 3 & 1.09 & 0.209 & 1.39 & 0.044 & -1.26 & 0.073 \\
\hline Collagen process & P4hal & 18451 & $\begin{array}{l}\text { Procollagen-proline 4-hydroxylase alpha } 1 \\
\text { polypeptide }\end{array}$ & 1.27 & 0.038 & 1.32 & 0.000 & -1.00 & 0.904 \\
\hline Collagen process & Loxl1 & 16949 & Lysyl oxidase-like 1 & 1.25 & 0.064 & 1.31 & 0.005 & 1.00 & 0.942 \\
\hline Collagen process & Pcolce & 18542 & $\begin{array}{l}\text { Procollagen C-endopeptidase enhancer } \\
\text { protein }\end{array}$ & 1.22 & 0.082 & 1.36 & 0.017 & -1.12 & 0.332 \\
\hline $\begin{array}{r}\text { Proteolysis } \\
\text { inhibitor }\end{array}$ & Timp1 & 21857 & Tissue inhibitor of metalloproteinase 1 & 1.05 & 0.595 & 1.35 & 0.038 & -1.22 & 0.188 \\
\hline $\begin{array}{r}\text { Proteolysis } \\
\text { inhibitor }\end{array}$ & Timp4 & 110595 & Tissue inhibitor of metalloproteinase 4 & 1.67 & 0.010 & 1.28 & 0.006 & 1.03 & 0.607 \\
\hline
\end{tabular}

The expression, fold change (FC) and $t$-test $p$ value $(p)$ were calculated from microarray analysis $(n=5)$

(Bmp1) was not changed, the upregulation of Pcolce (Fig. 2) could lead to an increase in proteinase activity and thus contribute significantly to the increased amount of C-peptides in extracellular medium.

\section{Discussion}

We used transcriptomics and proteomics to reveal the effects of insulin on adipocyte secretion. From a proteomics view, insulin had a significant impact on protein secretion. However, transcription is not the major regulation point for these secreted proteins. A discrepancy between mRNA and protein levels has been observed in other studies [18, 19]. In addition to technical differences, the proposed molecular explanations include polysome activation on mRNA, alternative mRNA splicing, protein turnover, specific proteolytic processing and
PTMs [20]. Further, the discrepancy between transcript and protein level largely depends on the nature of the proteins studied. Maturation of the secreted proteins involves significant PTMs. For example, glycosylation and signal peptide cleavage are common events for these proteins. A secreted protein undergoes synthesis, modification, and transport before it can be located to its destined extracellular space. Therefore, it is reasonable to expect a low correlation between its mRNA and secreted protein level.

Here, we go further and propose a biological role for this discrepancy in the action of insulin. Insulin is a very potent hormone that is precisely regulated both spatially and temporally [21]. A highly effective means of regulating the activity of target proteins involved in insulin signalling pathways is phosphorylation, one form of PTM. Likewise, PTM can also be used to efficiently promote the secretion of proteins, as an alternative to upregulation of their 
Table 2 Expression of secreted proteins regulated by insulin

\begin{tabular}{|c|c|c|c|c|c|c|c|c|c|}
\hline \multirow[b]{3}{*}{ Protein description } & \multicolumn{4}{|c|}{ Protein secretion level } & \multirow[b]{3}{*}{ Gene } & \multicolumn{4}{|c|}{ mRNA level } \\
\hline & \multicolumn{2}{|l|}{ Ins/basal } & \multicolumn{2}{|c|}{ Ins + Glc/Glc } & & \multicolumn{2}{|c|}{ Ins/basal } & \multicolumn{2}{|c|}{ Ins + Glc/Glc } \\
\hline & Overall & $\begin{array}{l}\text { Max } \\
\text { FC }\end{array}$ & Overall & $\begin{array}{l}\text { Max } \\
\text { FC }\end{array}$ & & $\mathrm{FC}$ & $p$ & $\mathrm{FC}$ & $p$ \\
\hline \multicolumn{10}{|l|}{ Downregulated proteins } \\
\hline Vimentin fragment & - & -4.6 & - & -13.9 & $\operatorname{Vim}$ & 1.17 & 0.016 & 1.11 & 0.187 \\
\hline Beta actin fragment & - & -7.6 & - & -6.8 & $A c t b$ & -1.12 & 0.077 & 1.20 & 0.028 \\
\hline Cofilin, non-muscle isoform & - & -1.9 & \pm & -1.6 & $C f l 1$ & 1.03 & 0.597 & 1.24 & 0.032 \\
\hline Gesolin fragment & \pm & -1.5 & - & 3.9 & Gsn & 1.10 & 0.236 & 1.11 & 0.115 \\
\hline Adiponectin & - & -2.1 & \pm & -1.5 & $A c d c$ & -1.12 & 0.320 & -1.03 & 0.852 \\
\hline ATP synthase D chain, mitochondrial & - & -2.1 & - & -2.9 & Atp $5 h$ & -1.24 & 0.861 & 1.03 & 0.744 \\
\hline Galectin-1 & - & -1.9 & - & -2.9 & Lgals 1 & -1.03 & 0.937 & 1.01 & 0.881 \\
\hline Peroxiredoxin 1 & - & -8.5 & - & -6.6 & $\operatorname{Prdx} 1$ & 1.09 & 0.241 & 1.03 & 0.801 \\
\hline Prohibitin & - & -4.0 & - & -5.0 & $P h b$ & -1.07 & 0.392 & 1.08 & 0.437 \\
\hline \multicolumn{10}{|l|}{ Upregulated proteins } \\
\hline Collagen I alpha 1 fragment and C-terminal peptide & + & 31.9 & + & 51.0 & Collal & 1.11 & 0.442 & 1.23 & 0.160 \\
\hline Collagen I alpha 2 fragment and C-terminal peptide & + & 14.7 & + & 14.2 & Colla2 & 1.05 & 0.596 & 1.20 & 0.132 \\
\hline Collagen III alpha 1 fragment and C-terminal peptide & + & 3.4 & + & 7.0 & Col3al & 1.17 & 0.170 & 1.16 & 0.134 \\
\hline Adipsin & + & 6.0 & + & 3.6 & $A d n$ & -1.20 & 0.204 & -1.11 & 0.397 \\
\hline Collagen V alpha 1 fragment & + & 3.5 & + & 4.8 & Col5al & 1.11 & 0.290 & 1.32 & 0.018 \\
\hline Matrix metalloproteinase-2 & + & 11.7 & \pm & 2.3 & Mmp2 & 1.17 & 0.502 & 1.31 & 0.102 \\
\hline Procollagen C-endopeptidase enhancer protein & + & 4.2 & + & 7.3 & Pcolce & 1.22 & 0.082 & 1.36 & 0.017 \\
\hline Pigment epithelium-derived factor fragment & + & 3.2 & + & 1.9 & Serpinf1 & 1.19 & 0.334 & 1.14 & 0.400 \\
\hline Heterogeneous nuclear 1 ribonucleoprotein A2/B1 & \pm & 1.2 & + & $\propto$ & Hnrpa2b1 & 1.02 & 0.928 & 1.00 & 0.964 \\
\hline Superoxide dismutase 3 , extracellular & \pm & -1.1 & + & 2.2 & Sod3 & 1.07 & 0.473 & 1.38 & 0.040 \\
\hline Secreted acidic cysteine-rich glycoprotein & + & 7.0 & + & 4.2 & Sparc & 1.03 & 0.623 & 1.18 & 0.076 \\
\hline Metalloproteinase inhibitor 2 & \pm & -1.1 & + & $\propto$ & Timp2 & -1.04 & 0.825 & 1.23 & 0.065 \\
\hline \multicolumn{10}{|l|}{ Possible downregulated proteins } \\
\hline Annexin A5, Coatomer protein subunit epsilon & - & -3.1 & - & -4.0 & Anxa5 & 1.02 & 0.813 & 1.04 & 0.893 \\
\hline (mixed with collagen III alpha 1 C-peptide) & & & & & Cope & 1.14 & 0.180 & 1.11 & 0.362 \\
\hline \multicolumn{10}{|l|}{ Possible upregulated proteins } \\
\hline \multirow{2}{*}{$\begin{array}{l}\text { Protein-lysine 6-oxidase, alpha enolase fragment } \\
\text { (mixed with collagen I alpha 2) }\end{array}$} & + & $\propto$ & + & $\propto$ & Lox & 1.07 & 0.763 & -1.04 & 0.787 \\
\hline & + & $\propto$ & + & $\propto$ & Enol & 1.20 & 0.079 & 1.21 & 0.157 \\
\hline \multirow{2}{*}{$\begin{array}{l}\text { Macrophage migration inhibitory factor } \\
\text { (mixed with beta-2 microglobulin) }\end{array}$} & + & 3.3 & + & 2.7 & Mif & 1.18 & 0.106 & 1.35 & 0.025 \\
\hline & & & & & $B 2 m$ & -1.01 & 0.902 & 1.02 & 0.879 \\
\hline $\begin{array}{l}\text { Nascent polypeptide associated complex alpha } \\
\text { (mixed with collagen V fragment) }\end{array}$ & + & 3.5 & + & 4.8 & $\mathrm{Naca}$ & \multicolumn{4}{|c|}{ undetectable signal on array } \\
\hline Nidogen (mixed with collagen I and VI) & $+(1 \mathrm{D})$ & & $+(1 \mathrm{D})$ & & Nidl & 1.04 & 0.529 & 1.09 & 0.293 \\
\hline \multicolumn{10}{|l|}{ Dual-regulated proteins } \\
\hline \multirow[t]{2}{*}{ Complement $\mathrm{C} 3$ beta chain } & + & 3.7 & + & 4.0 & $C 3$ & 1.10 & 0.191 & 1.13 & 0.223 \\
\hline & - & -2.3 & - & -7.2 & & & & & \\
\hline
\end{tabular}

Protein secretion levels were measured by $2 \mathrm{D}$ gel analysis $(n=3)$ or by $1 \mathrm{D}$ analysis $(1 \mathrm{D})$ when indicated. When more than one spot had the same protein identification, the total signal of all spots was used for comparison to show the overall change (+: increased, $\pm:$ no change, $-:$ decreased) and the fold change of the spot with the maximum change is given (Max FC). mRNA levels were measured by microarray analysis $(n=5)$. $F C$, fold change; $\alpha$, spot appeared only in condition with insulin. $p$ values are for $t$-test

transcription. On the basis of our findings, insulin promotes collagen protein secretion via the transcriptional upregulation of enzymes involved in PTMs. Via processing enzymes, this regulation can evoke a highly amplified effect. For example PCOLCE, which was up-regulated in this study, can enhance the activity of procollagen Cendopeptidase up to 20 -fold [17].
Besides an effect on collagens, increased processing and other PTMs might also explain effects on other secreted proteins, such as adipsin, SPARC and complement $\mathrm{C} 3$, which are highly upregulated by insulin at the secretion level, but not at the mRNA level. Alternatively, this could be explained by a general upregulation of exocytosis. 
Fig. 4 Collagen $\mathrm{C}$ peptides as measured by $2 \mathrm{D}$ gel analysis in (a) basal, (b) high glucose, (c) high insulin and (d) high insulin high glucose conditions. A section of one representative $2 \mathrm{D}$ gel image per condition is shown. Circles, collagen I alpha 1 C-peptide; squares, collagen III alpha 1 C-peptide; diamonds, collagen I alpha 2 C-peptide a

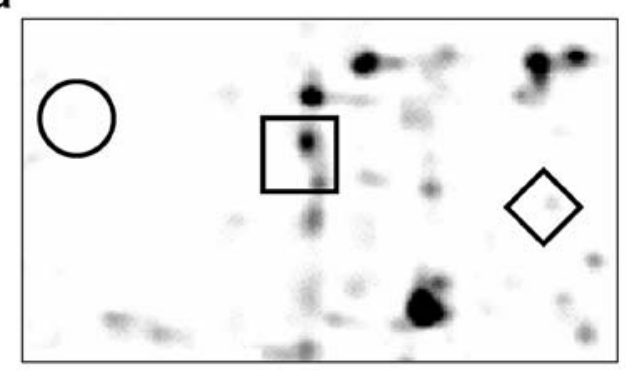

b

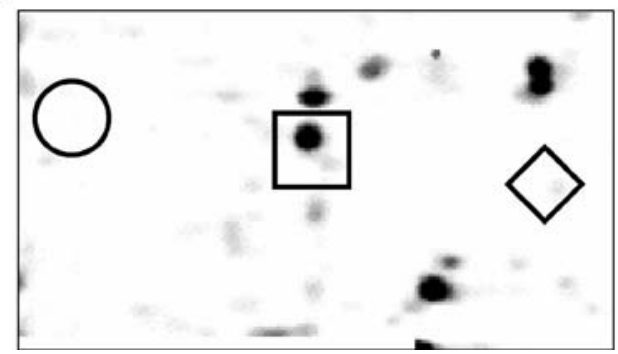

C

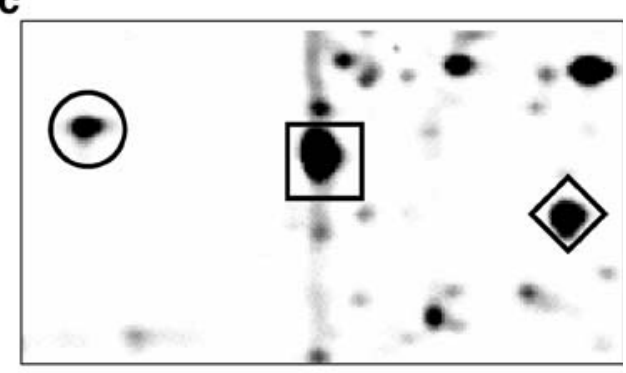

d

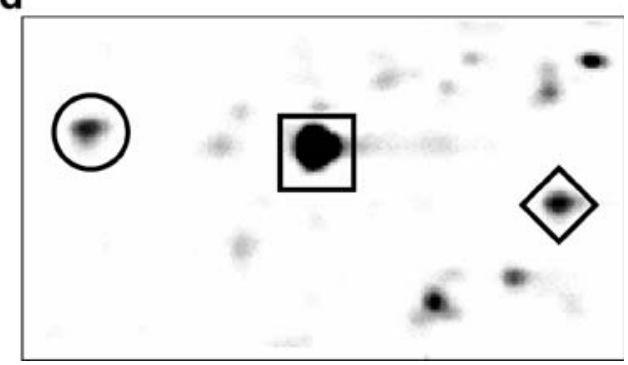

Using the 2D gel system to study adipocyte secretion in mouse 3T3-L1 cells has several advantages and limitations, as discussed previously [10]. Only part of the secretory proteome is visualised. For instance, we were not able to cover all known adipocyte-secreted proteins to address their response to insulin and determine the relationship between their mRNA and secretion levels. Further, the change of protein quantity based on a single spot may be underestimated if a protein is present in several isoforms and thus as several spots on the gel, or overestimated if one gel spot contains more than one protein. Therefore we took all the spots with the same identification into account and provide information on mixed spots in Table 2. One major advantage of $2 \mathrm{D}$ gel analysis is that it is useful for visualising protein modifications. The different isoforms

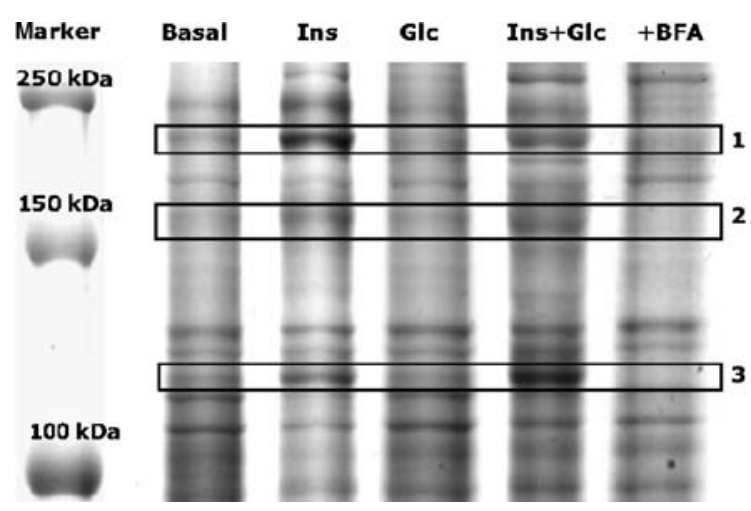

Fig. 5 Differentially expressed high-molecular-weight proteins as measured by $1 \mathrm{D}$ gel analysis. One representative image is shown. A sample treated with brefeldin A (+BFA) was used to verify secretion. Band 1: procollagen I alpha 1+ procollagen III alpha 1; band 2: nidogen+procollagen I alpha $2+$ collagen VI alpha 2; band 3: complement $\mathrm{C} 3$ and modifications can be separated on the basis of their different isoelectric points and mol. wt. The importance of processing and secondary modification as the effect of

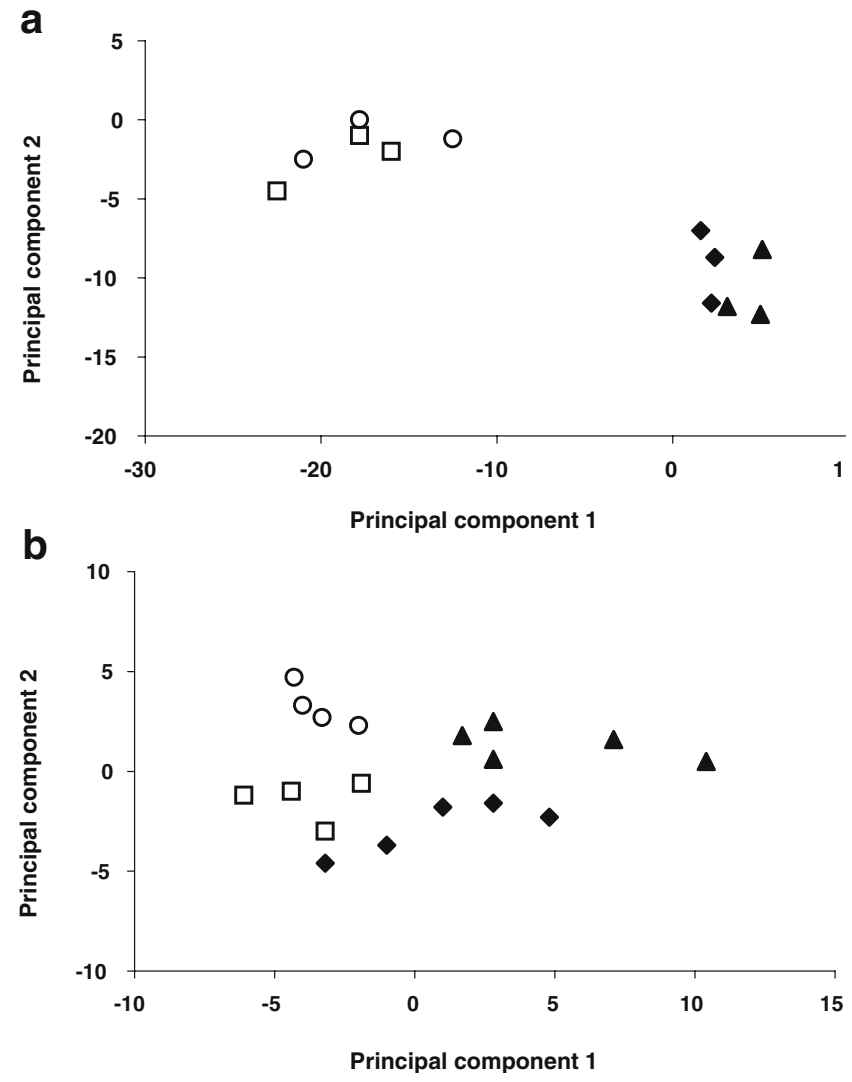

Fig. 6 Discriminant principal component analysis (a) of secreted proteins on the secretion pattern by $2 \mathrm{D}$ gel analysis, and (b) of matched mRNAs on the expression pattern by microarray analysis. One symbol represents one sample profile in the groups of basal $(\downarrow)$, insulin $(\square)$, glucose $(\boldsymbol{\Delta})$ or insulin + glucose $(\circ)$ 
insulin is illustrated by the $2 \mathrm{D}$ analysis of the C-peptides of collagens. Another example is that the beta chain of complement C3 had three spots on the gel [10]. Two spots were upregulated, as was the $\mathrm{C} 3$ complete chain on $1 \mathrm{D}$ gel, and one spot was downregulated by insulin, indicating different modifications. Detailed information about the modifications may provide more insight into the exact effect of insulin on the expression and function of these proteins.

Insulin promotes cell survival and anti-apoptosis [22, 23]. Numerous studies have shown that cell survival and death strongly depend on cell adhesion and the ECM [24, 25]. Our previous work showed that ECM components represent a major part of the proteins secreted from 3T3-L1 adipocytes in vitro [10]. We propose that the upregulation of secretion of ECM components, together with the increased gene expression of adhesion molecules (Table 1) by insulin, contributes to a strengthened survival of in vitro cultured adipocytes. Since in vivo adipocytes are intensively supported by the basement membrane [26], insulin may similarly affect adipocyte survival in vivo.

Apart from adiponectin and galectin, the downregulated proteins in the medium are not genuine secreted proteins. As a reflection of secreted proteinase activity, their presence in the medium may be caused by leakage and shedding [10]. In view of the improved cell survival, it is not surprising to see a decrease of leaked cellular proteins. The difference from other genuine secreted proteins implies that secretion of adiponectin and galectin 1 is regulated by insulin in a different way. This may be consistent with the finding that adiponectin and collagen VI alpha 3 are in two distinct exocytosis compartments [7].

Here, we report for the first time that Sulf2 expression is significantly upregulated by insulin in 3T3-L1 adipocytes in vitro. Sulf2 belongs to the family of extracellular endosulfatases that are able to remodel the 6-O sulfation state of cell surface heparan sulfate proteoglycans (HSPG). Again, its regulation shows that transcription of PTM enzyme genes is a target of insulin in adipocytes. The human SULF2 (KIAA1247) gene is located in a type 2 diabetes susceptibility region on human chromosome 20 [27]. HSPG has been implicated in diverse physiological processes [28-31] by binding ligands for which the specific structure and/or negative charge of the heparan sulfate chain is instrumental [32]. It has already been reported that de-sulfation of HSPG accounts for altered function. For example, avian QSULF1, a family member of Sulf2, inhibited angiogenesis in vitro [33]. Analogously, desulfation triggered by insulin via Sulf2 may induce functional changes in adipocytes. Interestingly, reduced amounts and an altered function of HSPG have been extensively reported in patients with type 2 and also type 1 diabetes $[34,35]$. The decrease of HSPG and their sulfation in diabetes may be due to a decrease of $N$-deacetylase [36], possibly induced by a high glucose level [37]. However, others have shown that high glucose only diminished the level of HSPG without significant changes in $N$-deacetylase and sulfation (especially N-sulfation) in cultured 3T3-L1 adipocytes [38]. Besides glucose, insulin has also been considered to underlie alteration of HSPG. The ${ }^{35} \mathrm{~S}$ labelled HSPG from liver were lower in obese and insulin-resistant Zucker rats than in their unaffected littermates [39]. Our results suggest that besides glucose, insulin might independently induce a pattern change in HSPG in cultured adipocytes. Immunocytochemical analysis with the antibody 10E4 (Seikagaku, Tokyo, Japan) has been used to assay the 6-O de-sulfation by transfected Qsulf1 in 3T3-L1 fibroblasts [40]. Using this antibody, we failed to detect a significant decrease of HSPG sulfation in 3T3-L1 mature adipocytes after treatment with insulin (data not shown). However, the $10 \mathrm{E} 4$ antibody may not be able to properly recognise 6-O sulfation [41].

In conclusion, we used a combined transcriptomic and proteomic approach to study the effect of insulin on mature 3T3-L1 adipocytes focusing on secreted proteins. Interestingly, we observed that insulin has a large effect on secretion but not on transcription of secreted protein genes. This discrepancy may be partly explained by the transcriptional upregulation of processing protein genes. It reveals that PTM is induced by insulin not only in signal transduction, but also in the regulation of secretion. Determination of quantity and activity of the PTM-enzymes will further support this hypothesis. Our study is one of the first to use transcriptomics and proteomics, and clearly demonstrates the added value of a combined genomics approach to the study of biological mechanisms.

Acknowledgements We thank our colleagues in the Functional Genomics Group and the Food Bioactives Group for technical support and helpful discussions. We also thank J. Broers and F. Houben (Department of Molecular Cell Biology) for help with immunocytochemistry. This study was financed by the Centre for Human Nutrigenomics in the Netherlands.

\section{References}

1. Kahn BB, Flier JS (2000) Obesity and insulin resistance. J Clin Invest 106:473-481

2. Fasshauer M, Paschke R (2003) Regulation of adipocytokines and insulin resistance. Diabetologia 46:1594-1603

3. Lafontan M (2005) Fat cells: afferent and efferent messages define new approaches to treat obesity. Annu Rev Pharmacol Toxicol 45:119-146

4. Faraj M, Lu HL, Cianflone K (2004) Diabetes, lipids, and adipocyte secretagogues. Biochem Cell Biol 82:170-190

5. Summers SA, Whiteman EL, Birnbaum MJ (2000) Insulin signaling in the adipocyte. Int J Obes Relat Metab Disord 24 (Suppl 4):S67-S70 
6. Lin Y, Berg AH, Iyengar P et al (2005) The hyperglycemiainduced inflammatory response in adipocytes: the role of reactive oxygen species. J Biol Chem 280:4617-4626

7. Bogan JS, Lodish HF (1999) Two compartments for insulinstimulated exocytosis in 3T3-L1 adipocytes defined by endogenous ACRP30 and GLUT4. J Cell Biol 146:609-620

8. Millar CA, Meerloo T, Martin S et al (2000) Adipsin and the glucose transporter GLUT4 traffic to the cell surface via independent pathways in adipocytes. Traffic 1:141-151

9. Chen X, Cushman SW, Pannell LK, Hess S (2005) Quantitative proteomic analysis of the secretory proteins from rat adipose cells using a 2D liquid chromatography-MS/MS approach. J Proteome Res 4:570-577

10. Wang P, Mariman E, Keijer J et al (2004) Profiling of the secreted proteins during 3T3-L1 adipocyte differentiation leads to the identification of novel adipokines. Cell Mol Life Sci 61:24052417

11. Pellis L, Franssen-van Hal NL, Burema J, Keijer J (2003) The intraclass correlation coefficient applied for evaluation of data correction, labeling methods, and rectal biopsy sampling in DNA microarray experiments. Physiol Genomics 16:99-106

12. Van Der Meer-Van Kraaij C, Van Lieshout EM, Kramer E, Van Der Meer R, Keijer J (2003) Mucosal pentraxin (Mptx), a novel rat gene 10 -fold down-regulated in colon by dietary heme. FASEB J 17:1277-1285

13. Bouwman F, Renes J, Mariman E (2004) A combination of protein profiling and isotopomer analysis using matrix-assisted laser desorption/ionization-time of flight mass spectrometry reveals an active metabolism of the extracellular matrix of 3T3L1 adipocytes. Proteomics 4:3855-3863

14. Ueki K, Fruman DA, Yballe CM et al (2003) Positive and negative roles of $\mathrm{p} 85$ alpha and $\mathrm{p} 85$ beta regulatory subunits of phosphoinositide 3-kinase in insulin signaling. J Biol Chem 278:48453-48466

15. Alberts B, Johnson A, Lewis J, Raff M, Roberts K, Walter P (2002) Cell junctions, cell adhesion, and the extracellular matrix. In: Gibbs S (ed) Molecular biology of the cell. Garland, New York, pp 1065-1126

16. Kivirikko KI, Helaakoski T, Tasanen K et al (1990) Molecular biology of prolyl 4-hydroxylase. Ann N Y Acad Sci 580: $132-142$

17. Ricard-Blum S, Bernocco S, Font B et al (2002) Interaction properties of the procollagen $\mathrm{C}$-proteinase enhancer protein shed light on the mechanism of stimulation of BMP-1. J Biol Chem 277:33864-33869

18. Chen G, Gharib TG, Huang CC et al (2002) Discordant protein and mRNA expression in lung adenocarcinomas. Mol Cell Proteomics 1:304-313

19. Griffin TJ, Gygi SP, Ideker T et al (2002) Complementary profiling of gene expression at the transcriptome and proteome levels in Saccharomyces cerevisiae. Mol Cell Proteomics 1: 323-333

20. Hegde PS, White IR, Debouck C (2003) Interplay of transcriptomics and proteomics. Curr Opin Biotechnol 14:647-651

21. Saltiel AR, Pessin JE (2002) Insulin signaling pathways in time and space. Trends Cell Biol 12:65-71

22. Kido Y, Nakae J, Accili D (2001) Clinical review 125: the insulin receptor and its cellular targets. J Clin Endocrinol Metab 86: 972-979
23. Barthel A, Schmoll D, Unterman TG (2005) FoxO proteins in insulin action and metabolism. Trends Endocrinol Metab 16:183-189

24. Santini MT, Rainaldi G, Indovina PL (2000) Apoptosis, cell adhesion and the extracellular matrix in the three-dimensional growth of multicellular tumor spheroids. Crit Rev Oncol Hematol 36:75-87

25. Farrelly N, Lee YJ, Oliver J, Dive C, Streuli CH (1999) Extracellular matrix regulates apoptosis in mammary epithelium through a control on insulin signaling. J Cell Biol 144:1337-1348

26. Pierleoni C, Verdenelli F, Castellucci M, Cinti S (1998) Fibronectins and basal lamina molecules expression in human subcutaneous white adipose tissue. Eur J Histochem 42:183-188

27. Fossey SC, Mychaleckyj JC, Pendleton JK et al (2001) A highresolution 6.0-megabase transcript map of the type 2 diabetes susceptibility region on human chromosome 20. Genomics 76:45-57

28. Bernfield M, Gotte M, Park PW et al (1999) Functions of cell surface heparan sulfate proteoglycans. Annu Rev Biochem 68:729-777

29. Iozzo RV (2001) Heparan sulfate proteoglycans: intricate molecules with intriguing functions. J Clin Invest 108:165-167

30. Wilsie LC, Chanchani S, Navaratna D, Orlando RA (2005) Cell surface heparan sulfate proteoglycans contribute to intracellular lipid accumulation in adipocytes. Lipids Health Dis 4:2

31. Mahley RW, Ji ZS (1999) Remnant lipoprotein metabolism: key pathways involving cell-surface heparan sulfate proteoglycans and apolipoprotein E. J Lipid Res 40:1-16

32. Nakato H, Kimata K (2002) Heparan sulfate fine structure and specificity of proteoglycan functions. Biochim Biophys Acta 1573:312-318

33. Wang S, Ai X, Freeman SD et al (2004) QSulf1, a heparan sulfate 6-O-endosulfatase, inhibits fibroblast growth factor signaling in mesoderm induction and angiogenesis. Proc Natl Acad Sci USA 101:4833-4838

34. Kozma EM, Olczyk K, Glowacki A et al (1996) Glycosaminoglycans of human serum and their alterations in diabetes mellitus. Acta Biochim Pol 43:567-574

35. Conde-Knape K (2001) Heparan sulfate proteoglycans in experimental models of diabetes: a role for perlecan in diabetes complications. Diabetes Metab Res Rev 17:412-421

36. Kjellen L, Bielefeld D, Hook M (1983) Reduced sulfation of liver heparan sulfate in experimentally diabetic rats. Diabetes 32: 337-342

37. Kofoed-Enevoldsen A, Noonan D, Deckert T (1993) Diabetes mellitus induced inhibition of glucosaminyl $N$-deacetylase: effect of short-term blood glucose control in diabetic rats. Diabetologia 36:310-315

38. Parthasarathy N, Gotow LF, Bottoms JD et al (2000) Influence of glucose on production and $\mathrm{N}$-sulfation of heparan sulfate in cultured adipocyte cells. Mol Cell Biochem 213:1-9

39. Olsson U, Egnell AC, Lee MR et al (2001) Changes in matrix proteoglycans induced by insulin and fatty acids in hepatic cells may contribute to dyslipidemia of insulin resistance. Diabetes 50:2126-2132

40. Ai X, Do AT, Lozynska O, Kusche-Gullberg M, Lindahl U, Emerson CP Jr (2003) QSulf1 remodels the 6-O sulfation states of cell surface heparan sulfate proteoglycans to promote Wnt signaling. J Cell Biol 162:341-351

41. van den Born J, Salmivirta K, Henttinen T et al (2005) Novel heparan sulfate structures revealed by monoclonal antibodies. J Biol Chem 280:20516-20523 